\title{
Chaperoning parasitism: the importance of molecular chaperones in pathogen virulence
}

\author{
UTPAL TATU ${ }^{1} *$ and $\mathrm{LEN} \mathrm{NECKERS}^{2}$ \\ ${ }^{1}$ Department of Biochemistry, Indian Institute of Science, Bangalore - 560012, India \\ ${ }^{2}$ Urologic Oncology Branch, Center for Cancer Research, National Cancer Institute, 9000 Rockville Pike, Bethesda, \\ MD 20892, USA
}

(Received 10 December 2013; revised 10 April 2014; accepted 15 April 2014)

Infectious organisms have to cope with demanding and rapidly changing environments during infection. This is particularly relevant for pathogens that utilize different hosts (e.g. insects and humans) to complete their life cycle. Other environmental challenges that may affect pathogen survival are often triggered in the host in response to infection. It is not surprising, therefore, that many successful pathogens not only co-opt the host chaperone machinery but also have developed their own robust chaperone systems to overcome the stresses associated with infection and propagation in multiple hosts.

During the past two decades much has been learned about the nature and function of the molecular chaperone heat shock protein 90 (Hsp90), especially concerning its association with several molecules and pathways important in maintaining normal cellular homoeostasis while also promoting cancer initiation, growth and survival. Hsp90 is an abundant $(2-5 \%$ of total cellular protein) molecular chaperone whose homoeostatic functions include stabilization and modulation of a number of proteins (clients) that comprise various cell signalling nodes, and fostering cellular responses to environmental stress (Picard, 2002; Wandinger et al. 2008; Taipale et al. 2010). The chaperone is 'hijacked', in what could be regarded as an 'addiction', by cancer cells, causing its expression to be elevated above that of non-transformed cells (Trepel et al. 2010). Like cancer, numerous pathogens have co-opted various components of the host cellular proteostasis machinery to support their infectivity and replication. Recently, molecular chaperones, including but not limited to Hsp90, have been identified as virulence factors in pathogenic infections. In the case of protozoan parasites, Hsp90 has been implicated in conversion from insect to host forms, in proliferation, and in drug resistance (Neckers and Tatu, 2008). Importantly, both the host and the parasite express

* Corresponding author: Department of Biochemistry, Indian Institute of Science, Bangalore-560012, India. E-mail: tatu@biochem.iisc.ernet.in
Hsp90 and related chaperone proteins, and both sources contribute to pathogen virulence and propagation.

Chaperone inhibitors, including Hsp90 inhibitors (see below), are generating interest for their utility in combating cancer resistance to other molecularly targeted agents. Indeed, inhibitors of Hsp90 have been proposed as candidate drugs for the treatments of diseases such as candidiasis and malaria, as well as trypanosomiasis (Banumathy et al. 2003; Pallavi et al. 2010). For example, Hsp90 inhibitors have been shown to reverse or delay the onset of tumour resistance to tyrosine kinase inhibitors, and resistance to antifungal drugs has been reversed in pre-clinical models of fungal infections (Neckers and Workman, 2012; Cowen, 2013; Miyajima et al. 2013). Preliminary data suggest that chaperone-directed therapy may similarly reduce viral fitness and counteract resistance to existing antiviral therapy (Geller et al. 2007). Moving forward, this represents one of the most promising clinical applications of chaperone therapeutics.

Canonical Hsp90 is found in all Kingdoms except Archaea (Large et al. 2009). In eukaryotes, including single-cell organisms, there are two Hsp90 isoforms: stress-inducible Hsp $90 \alpha$ and constitutively expressed Hsp90 $\beta$ (Grad et al. 2010). These isoforms, although highly homologous, do not fully complement each other. Hsp90 $\beta$ knock-out is embryologically lethal, while mice lacking $\mathrm{Hsp} 90 \alpha$ are viable although males are sterile (Voss et al. 2000). Higher eukaryotes also express organelle-specific Hsp90 paralogues. Glucose-regulated protein 94 (Grp94), also known as Hsp90B1, is found in the endoplasmic reticulum, where it participates in folding proteins destined for secretion or plasma membrane insertion (Dollins et al. 2007; Frey et al. 2007). Hsp75, also known as TNF receptor-associated protein 1 (TRAP1), is a mitochondrial paralogue that provides protection from proteotoxic stress and recently has been shown to directly modulate mitochondrial metabolism (Felts et al. 2000; Leskovar et al. 2008; Sciacovelli et al. 2013; Yoshida et al. 2013). 
Hsp90 exists as a homodimer and is a member of the ATPase/kinase GHKL (DNA Gyrase, Hsp90, Histidine Kinase, MutL) superfamily - a small group of proteins that are characterized by a unique ATP binding cleft that is unlike the ATP binding domains of most other proteins, including kinases (Picard, 2002; Pearl and Prodromou, 2006). The N terminal domain of each Hsp90 protomer contains the ATPbinding pocket, which is also the binding site for a number of highly specific ATP-competitive inhibitors that are now in clinical trial (Alarcon et al. 2012). The N-domain is also a site for co-chaperone interactions (see below). The middle (M) domain of Hsp90 has binding sites for clients and additional co-chaperones, and the C-terminal domain contains the dimerization motif as well as binding sites for other co-chaperones. Connecting the $\mathrm{N}$ and $\mathrm{M}$ domains are a number of charged amino acids. This unstructured region, that is highly divergent in both length and sequence among Hsp90 proteins of different species (in contrast to the rest of the protein, which contains large regions that are highly homologous between species), is referred to as the 'charged linker' and plays an essential, although as yet not completely understood role in Hsp90 chaperone function (Hainzl et al. 2009; Tsutsumi et al. 2009, 2012).

In all organisms, Hsp90 is a dynamic protein that undergoes a conformational cycle, critical to its chaperone function, whose directionality is determined in large part by ATP binding/hydrolysis and by co-chaperone binding. However, the Hsp90-directed chaperone cycle is complex and its regulation is also impacted by numerous posttranslational modifications to Hsp90 and the various co-chaperones (Mollapour and Neckers, 2011). Clinically evaluated Hsp90 inhibitors, including those that are discussed in the articles in this Special Issue, disrupt the chaperone cycle by displacing ATP and occupying the ATP binding pocket, which is highly homologous between species. In the absence of ATP binding, Hsp90 becomes nonfunctional and many of its clients become ubiquitinated and ultimately degraded. While not completely understood, this is a regulated process that requires client dissociation from Hsp90 and concomitant association with an Hsp70 chaperone complex that also contains one or more E3 ubiquitin ligases (Neckers, 2006; Pearl and Prodromou, 2006; Trepel et al. 2010).

Co-chaperones interact with distinct Hsp90 conformational states. Presumably, these interactions lower the energy barrier between certain conformations while stabilizing other conformations, thus helping to provide directionality to and modulate the timing of the Hsp90 cycle (Hessling et al. 2009; Mickler et al. 2009). Certain co-chaperones, such as Hop and Cdc37, stabilize an ATPase-incompetent Hsp90 conformation while simultaneously assisting in delivery of distinct sets of client proteins (steroid hormone receptors and kinases, respectively) to the Hsp90 chaperone machine. Other co-chaperones, such as p23, stabilize an ATPase competent Hsp90 conformation while reducing ATP hydrolysis, thereby increasing client protein dwell time on Hsp90 (important for hard-to-fold clients). Aha1 is a cochaperone that facilitates the energy-demanding conformational change to establish Hsp90 ATPase competence and to drive the chaperone cycle to completion. Concomitant with ATP hydrolysis, client proteins are released from the Hsp90 chaperone complex. Most Hsp90 co-chaperones have been identified in infectious pathogens and they likely modulate Hsp90 in these organisms somewhat similarly to their role(s) in higher eukaryotes. These complex and highly regulated conformational dynamics allow Hsp90 to bind, chaperone and release client proteins at a rate that is tailored to the needs of individual clients. During the chaperone process, the conformation and activity/stability of the client protein is altered (McLaughlin et al. 2004; Shiau et al. 2006; Vaughan et al. 2006; Hessling et al. 2009; Mickler et al. 2009).

Analysis of Hsp90 sequences and higher order structures across the evolutionary ladder reveals an interesting parallel between Hsp90 structures and complexity of species. While Archaebacteria do not show the presence of Hsp90, eubacteria carry a full-length Hsp90 primary structure showing the fully developed domain structures described above. Further, as we look at the Hsp90 in lower eukaryotes, one finds the presence of accessory factors or co-chaperones that can modulate Hsp90 ATPase and thereby their client interactions. While early eukaryotes, which include many protozoan parasites, possess only a few co-chaperone partners, higher eukaryotes show the presence of a full complement of co-chaperone repertoire. Importantly it appears that the jump from a minimalist Hsp90 chaperone such as HtpG to a multi-chaperone complex may have coincided with the development of parasitism (see Fig. 1). There appears to be an important link between parasite chaperones and the adaptation of these parasites to the host cell environments.

Among other evolutionary forces, demands of the daunting host cell milieu may have played an important role in shaping the biochemical activities, interactomes and cellular localizations of parasite chaperones. Chaperones, in turn, may have influenced not only the virulence of these parasites but also their speciation. It appears that the evolution of molecular chaperones and parasitism may have progressed hand-in-hand. As a corollary to the above arguments, it appears plausible that chaperones may have value as targets for the treatment of infectious diseases.

The articles in this Special Issue of Parasitology describe the current state of knowledge of the role(s) 

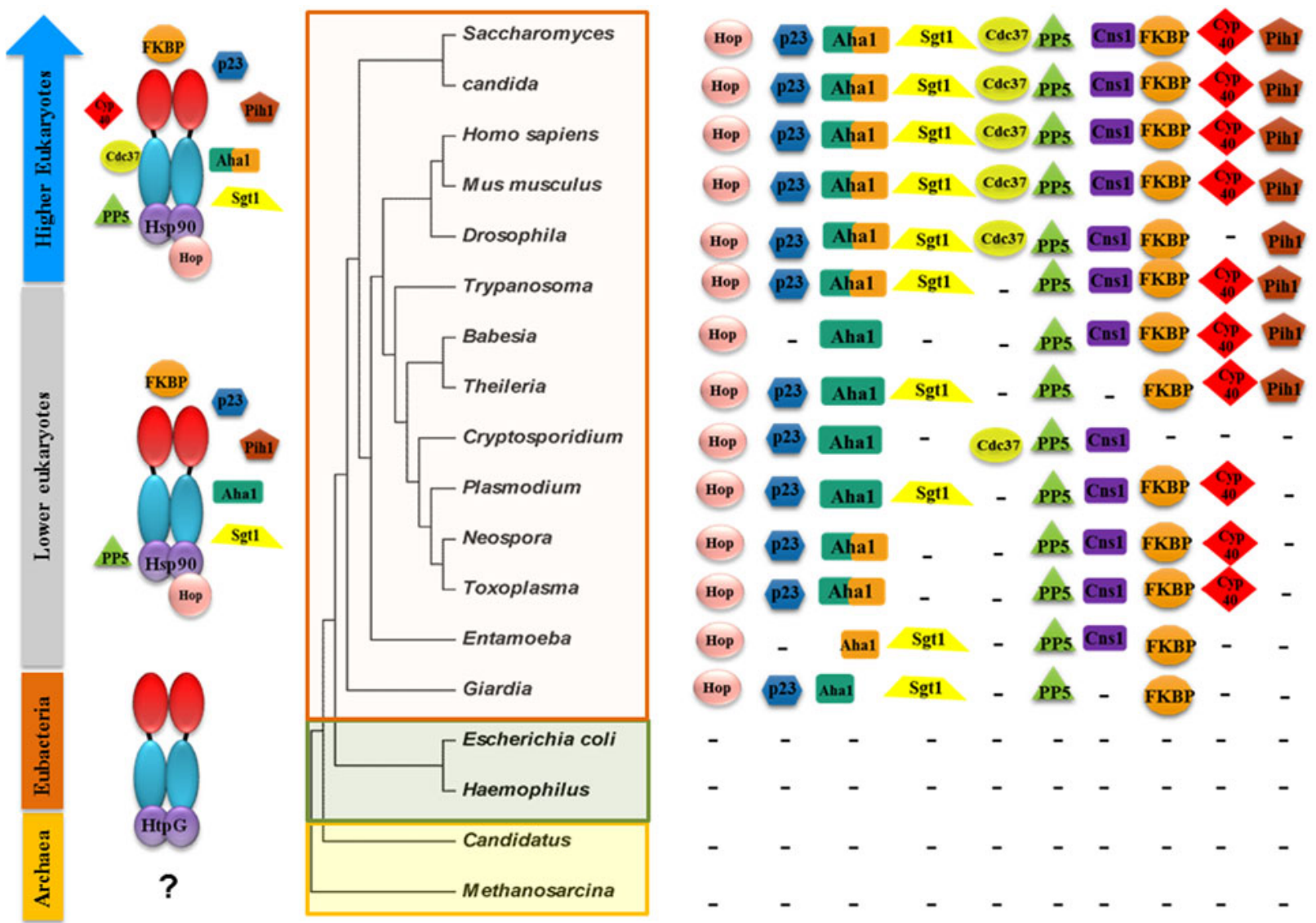

Fig. 1. The evolution of the Hsp90 multi-chaperone complex marks the development of complex species. The status of Hsp90 and its co-chaperone repertoire in different organisms along the evolutionary ladder is shown. While

Archaebacteria seem to lack a discernible full-length Hsp90, eubacteria possess a minimalist htpG-like Hsp90 devoid of co-chaperone modulators. The presence of rudimentary co-chaperones is seen from early branching eukaryotes (many of which were parasites) to higher eukaryotes possessing a full complement of co-chaperone repertoire.

of Hsp90 and related chaperone proteins in promoting pathogenic virulence, as well as the prospects of combating infection by targeting these 'housekeeping' proteins. By simultaneously targeting both the parasite and the host, such a treatment strategy may provide direct therapeutic benefit while also reducing parasite fitness and ability to evade other therapeutic interventions. Angel, Figueras and Alomar et al. emphasize the potential role of Hsp90 in the essential cellular processes of Toxoplasma gondii as well as the analysis of the comparative experimental data of the chaperone in $T$. gondii, yeast and humans. A critical evaluation of the current and future perspectives of Plasmodial Hsp 40 and Hsp 70 chaperones is put forth by Pesce and Blatch in their review. This is further supported by an article by Bayih and Pillai which deals with the pre-clinical mouse trials of Hsp90 inhibitors in the treatment of malaria. The regulatory roles of the co-chaperones of Hsp90 in Plasmodium falciparum and their impact on the drug design of antimalarials are summarized by Chua, Low and Sim. The prospective role of the Hsp90 complex in signal transduction pathways in Leishmania species is discussed by Hombach and Clos. They also highlight the possibility of using protein kinases and critical phosphorylation sites as novel therapeutic targets. Veri and Cowen underline the prospects of using Hsp90 and its cochaperones as targets for the treatment of fungal infections like candidiasis. The role of chaperones like Hsp90 in the treatment of HIV is discussed by Low and Fassati. Human and veterinary infections caused by helminth pathogens and Trypanosoma species can also be treated by targeting Hsp90. The prospects of using Hsp90 inhibitors to treat helminths infections have been elaborated by Gillan and Devaney. Similarly, Rochani, Mitra and Tatu expound the use of Hsp90 as a target in the treatment of Trypanosoma infections in their article. As in the case of cancer, molecular chaperones, and Hsp90 in particular, are emerging as novel and exciting therapeutic targets to combat pathogenic infection.

\section{ACKNOWLEDGEMENTS}

UT would like to thank Ms Meetali Singh, Mr Rishikumar Nageshan and Ms D.T. Vishruta for helping with the preparation of Fig. 1 and critical review of the manuscript. Financial help from DBT and DST are gratefully acknowledged. 


\section{REFERENCES}

Alarcon, S. V., Mollapour, M., Lee, M. J., Tsutsumi, S., Lee, S., Kim, Y.S., Prince, T., Apolo, A. B., Giaccone, G., Xu, W. Neckers, L. M. and Trepe1, J. B. (2012). Tumor-intrinsic and tumorextrinsic factors impacting hsp90-targeted therapy. Current Molecular Medicine 12, 1125-1141.

Banumathy, G., Singh, V., Pavithra, S. R. and Tatu, U. (2003). Heat shock protein 90 function is essential for Plasmodium falciparum growth in human erythrocytes. Fournal of Biological Chemistry 278, 18336-18345. Cowen, L. (2013). The fungal Achilles' heel: targeting Hsp90 to cripple fungal pathogens. Current Opinion in Microbiology 16, 377-384.

Dollins, D. E., Warren, J. J., Immormino, R. M. and Gewirth, D. T. (2007). Structures of GRP94-nucleotide complexes reveal mechanistic differences between the Hsp90 chaperones. Molecular Cell 28, 41-56. doi: 10.1016/j.molcel.2007.08.024.

Felts, S. J., Owen, B. A., Nguyen, P., Trepel, J., Donner, D. B. and Toft, D. O. (2000). The hsp90-related protein TRAP1 is a mitochondrial protein with distinct functional properties. Fournal of Biological Chemistry 275, 3305-3312.

Frey, S., Leskovar, A., Reinstein, J. and Buchner, J. (2007). The ATPase cycle of the endoplasmic chaperone Grp94. Fournal of Biological Chemistry 282, 35612-35620. doi: 10.1074/jbc.M704647200.

Geller, R., Vignuzzi, M., Andino, R. and Frydman, J. (2007). Evolutionary constraints on chaperone-mediated folding provide an antiviral approach refractory to development of drug resistance. Genes and Development 21, 195-205

Grad, I., Cederroth, C. R., Walicki, J., Grey, C., Barluenga, S., Winssinger, N., De Massy, B., Nef, S. and Picard, D. (2010). The molecular chaperone Hsp90 alpha is required for meiotic progression of spermatocytes beyond pachytene in the mouse. PLoS One 5, e15770. doi: 10.1371/journal.pone.0015770.

Hainzl, O., Lapina, M. C., Buchner, J. and Richter, K. (2009). The charged linker region is an important regulator of Hsp 90 function. Fournal of Biological Chemistry 284, 22559-22567. doi: 10.1074/jbc.M109.031658.

Hessling, M., Richter, K. and Buchner, J. (2009). Dissection of the ATP-induced conformational cycle of the molecular chaperone Hsp90. Nature: Structural \& Molecular Biology 16, 287-293. doi: 10.1038/ nsmb. 1565 .

Large, A. T., Goldberg, M. D. and Lund, P. A. (2009). Chaperones and protein folding in the Archaea. Biochemical Society Transactions 37, 46-51. doi: 10.1042/BST0370046.

Leskovar, A., Wegele, H., Werbeck, N.D., Buchner, J. and Reinstein, J. (2008). The ATPase cycle of the mitochondrial Hsp90 analog Trap1. Fournal of Biological Chemistry 283, 11677-11688. doi: 10.1074/jbc. M709516200.

McLaughlin, S. H., Ventouras, L. A., Lobbezoo, B. and Jackson, S. E (2004). Independent ATPase activity of Hsp90 subunits creates a flexible assembly platform. Fournal of Molecular Biology 344, 813-826. doi: 10.1016/ j.jmb.2004.09.055.

Mickler, M., Hessling, M., Ratzke, C., Buchner, J. and Hugel, T. (2009). The large conformational changes of Hsp90 are only weakly coupled to ATP hydrolysis. Nature Structural \& Molecular Biology 16, 281-286. doi: 10.1038/nsmb.1557

Miyajima, N., Tsutsumi, S., Sourbier, C., Beebe, K., Mollapour, M. Rivas, C., Yoshida, S., Trepel, J., Huang, Y., Tatokoro, M., Shinohara, N., Nonomura, K. and Neckers, L. (2013). The HSP90 inhibitor ganetespib synergizes with the MET kinase inhibitor crizotinib in both crizotinib-sensitive and -resistant MET-driven tumor models. Cancer Research 73, 7022-7033.

Mollapour, M. and Neckers, L. (2011). Post-translational modification of Hsp90 and their contributions to chaperone regulation. Biochimica et Biophysica Acta 1823, 648-655. doi: S0167-4889(11)00217-5; [pii] 10.1016/j.bbamcr.2011.07.018
Neckers, L. (2006). Using natural product inhibitors to validate Hsp90 as a molecular target in cancer. Current Topics in Medicinal Chemistry $\mathbf{6}$, 1163-1171

Neckers, L. and Tatu, U. (2008). Molecular chaperones in pathogen virulence: emerging new targets for therapy. Cell Host and Microbe 4 519-527.

Neckers, L. and Workman, P. (2012). Hsp90 molecular chaperone inhibitors: are we there yet? Clinical Cancer Research 18, 64-76.

Pallavi, R., Roy, N., Nageshan, R. K., Talukdar, P., Pavithra, S. R., Reddy, R., Venketesh, S., Kumar, R., Gupta, A. K., Singh, R. K., Yadav, S. C. and Tatu, U. (2010). Heat shock protein 90 as a drug target against protozoan infections: biochemical characterization of HSP90 from Plasmodium falciparum and Trypanosoma evansi and evaluation of its inhibitor as a candidate drug. Fournal of Biological Chemistry $\mathbf{2 8 5}$ 37964-37975

Pearl, L. H. and Prodromou, C. (2006). Structure and mechanism of the Hsp90 molecular chaperone machinery. Annual Review of Biochemistry 75, 271-294. doi: 10.1146/annurev.biochem.75.103004.142738.

Picard, D. (2002). Heat-shock protein 90, a chaperone for folding and regulation. Cellular and Molecular Life Sciences 59, 1640-1648.

Sciacovelli, M., Guzzo, G., Morello, V., Frezza, C., Zheng, L., Nannini, N., Calabrese, F., Laudiero, G., Esposito, F. Landriscina, M., Defilippi, P., Bernardi, P. and Rasola, A. (2013) The mitochondrial chaperone TRAP1 promotes neoplastic growth by inhibiting succinate dehydrogenase. Cell Metabolism 17, 988-999.

Shiau, A. K., Harris, S. F., Southworth, D. R. and Agard, D. A. (2006) Structural analysis of E. coli Hsp90 reveals dramatic nucleotide-dependent conformational rearrangements. Cell 127, 329-340. doi: 10.1016/j. cell.2006.09.027.

Taipale, M., Jarosz, D. F. and Lindquist, S. (2010). HSP90 at the hub of protein homeostasis: emerging mechanistic insights. Nature Reviews. Molecular Cell Biology 11, 515-528. doi: 10.1038/nrm2918.

Trepel, J., Mollapour, M., Giaccone, G. and Neckers, L. (2010) Targeting the dynamic HSP90 complex in cancer. Nature Reviews. Cancer 10, 537-549. doi: 10.1038/nrc2887.

Tsutsumi, S., Mollapour, M., Graf, C., Lee, C. T., Scroggins, B. T., Xu, W., Haslerova, L., Hessling, M., Konstantinova, A. A. Trepel, J. B., Panaretou, B., Buchner, J., Mayer, M.P. Prodromou, C. and Neckers, L. (2009). Hsp90 charged-linker truncation reverses the functional consequences of weakened hydrophobic contacts in the N domain. Nature Structural \& Molecular Biology 16, 1141-1147. doi: $10.1038 / \mathrm{nsmb} .1682$

Tsutsumi, S., Mollapour, M., Prodromou, C., Lee, C. T., Panaretou, B., Yoshida, S., Mayer, M. P. and Neckers, L. M. (2012). Charged linker sequence modulates eukaryotic heat shock protein 90 (Hsp90) chaperone activity. Proceedings of the National Academy of Sciences USA 109, 2937-2942. doi: 10.1073/pnas.1114414109.

Vaughan, C. K., Gohlke, U., Sobott, F., Good, V. M., Ali, M. M., Prodromou, C., Robinson, C. V., Saibil, H. R. and Pearl, L. H. (2006) Structure of an Hsp90-Cdc37-Cdk4 complex. Molecular Cell 23, 697-707. doi: 10.1016/j.molcel.2006.07.016.

Voss, A. K., Thomas, T. and Gruss, P. (2000). Mice lacking HSP90beta fail to develop a placental labyrinth. Development 127, 1-11

Wandinger, S. K., Richter, K. and Buchner, J. (2008). The Hsp90 chaperone machinery. Fournal of Biological Chemistry 283, 18473-18477. doi: 10.1074/jbc.R800007200.

Yoshida, S., Tsutsumi, S., Muhlebach, G., Sourbier, C., Lee, M. Lee, S., Vartholomaiou, E., Tatokoro, M., Beebe, K., Miyajima, N., Mohney, R., Chen, Y., Hasumi, H., Xu, W., Fukushima, H. Nakamura, K., Koga, F., Kihara, K., Trepel, J., Picard, D. and Neckers, L. (2013). Molecular chaperone TRAP1 regulates a metabolic switch between mitochondrial respiration and aerobic glycolysis. Proceedings of the National Academy of Sciences USA 110 E1604-E1612. 